\title{
Long-term variations in the heliosphere
}

Conference or Workshop Item

Accepted Version

Owens, M. J., Lockwood, M., Riley, P. and Barnard, L. (2018) Long-term variations in the heliosphere. In: IAU Symposium 340, 19-23 Feb 2018, Jaipur, India. Available at https://centaur.reading.ac.uk/78446/

It is advisable to refer to the publisher's version if you intend to cite from the work. See Guidance on citing.

Published version at: https://doi.org/10.1017/S1743921318000972

All outputs in CentAUR are protected by Intellectual Property Rights law, including copyright law. Copyright and IPR is retained by the creators or other copyright holders. Terms and conditions for use of this material are defined in the End User Agreement.

\section{www.reading.ac.uk/centaur}

\section{CentAUR}

Central Archive at the University of Reading

Reading's research outputs online 


\title{
Long-term variations in the heliosphere
}

\author{
Mathew J. Owens ${ }^{1}$ Mike Lockwood ${ }^{1}$ Pete Riley $^{2}$ and Luke Barnard ${ }^{1}$ \\ ${ }^{1}$ Department of Meteorology, University of Reading, Reading RG6 6BB, UK \\ email: $m$. j . owens@reading . ac.uk \\ ${ }^{2}$ Predictive Science Inc., 9990 Mesa Rim Rd, Suite 170, San Diego, CA 92121, USA
}

\begin{abstract}
Reconstructions of long-term solar variability underpin our understanding of the solar dynamo, potential tropospheric climate implications and future space weather scenarios. Prior to direct spacecraft measurements of the heliospheric magnetic field (HMF) and solar wind, accurate annual reconstructions are possible using geomagnetic and sunspot records. On longer timescales, information about the $\mathrm{HMF}$ can be extracted from cosmogenic radionuclide records, particularly ${ }^{14} \mathrm{C}$ in ancient trees and ${ }^{10} \mathrm{Be}$ in ice sheets. These proxies, and what they reveal about the HMF and solar wind, are briefly reviewed here.
\end{abstract}

Keywords. solar wind, heliosphere, solar-terrestrial relations, solar variability

\section{Introduction}

Long-term solar magnetic variability is of great interest for understanding stellar dynamos and for its terrestrial impacts, in terms of both space weather and space climate change, and possible changes in regional tropospheric climates. This paper aims to provide a broad overview of what we know about the heliosphere, and hence solar magnetic field, over centennial and millennial time scales (see Usoskin (2017); Owens and Forsyth (2013); Lockwood (2013) and references therein for more detail).

\section{In situ spacecraft observations}

The heliospheric magnetic field (HMF) and solar wind plasma can be measured directly by in situ spacecraft. Since 1964, there have been sufficient observations in near-Earth space to enable annual mean values of the HMF to be computed with uncertainty below the $10 \%$ level (Owens et al. 2016). These data form the OMNI dataset (King and Papitashvili 2005) and are summarised in Figure 1. There is a clear solar variation in the HMF as measured both by the local intensity, $|B|$, and the more global measure of total heliospheric magnetic flux (Owens et al. 2008). Annual means of solar wind speed, however, show very little variation in near-Earth space, though globally it is known to peak at solar minimum and reach a minimum at solar maximum (McComas et al. 2003).

\section{Heliospheric reconstructions}

To extend further into the past, it is necessary to use heliospheric proxies. The most direct proxy for conditions in near-Earth space is the geomagnetic record. Solar wind energy input to the magnetosphere varies with (in order of importance) the HMF intensity and direction, the solar wind speed, and the solar wind mass density (Vasyliunas et al. 1982). By using different geomagnetic indices with different solar wind sensitivities, it is possible to isolate the HMF and $V_{S W}$ contributions (Svalgaard and Cliver 2005; Lockwood et al. 2014). Due to high-frequency variations in the HMF orientation, these 
reconstructions can only be reliably made at the annual time resolution, over which geometric effects essentially average out (Lockwood 2013). For annual means, the agreement between geomagnetic reconstructions of $|B|$ and direct spacecraft measurements is very high. In fact, owing to the limited spacecraft sampling prior to 1996, the geomagnetic reconstructions of $|B|$ back to the 1964 have lower uncertainty than the spacecraft observations. Geomagnetic reconstructions have been reliably extended back to 1845 . By combining the local $|B|$ and $V_{S W}$ reconstructions and assuming a Parker spiral HMF, $\left|B_{R}\right|$ and hence the (global) total heliospheric flux can be computed (Lockwood et al. 2014). This is shown as the blue line in the bottom panel of Figure 2.

Prior to 1845, annual reconstructions are based on the telescopic sunspot record. Owing to the brightness variations associated with sunspots relative to the rest of the photosphere, total solar irradiance can be modelled using sunspot number, or preferably, sunspot area records (Krivova et al. 2007). While sunspots are a manifestation of the solar magnetic field, converting sunspot number to a quantitative estimate of the HMF requires a number of assumptions. Firstly, that sunspots are a proxy for the production rate of new heliospheric flux (Solanki et al. 2002). It may be surprising that sunspots, the footpoints of closed coronal magnetic flux, act as reliable proxies for flux "open" to the heliosphere. But the generation of new open solar flux can only occur by closed coronal loops rising through the source surface, which is driven by photospheric flux emergence in sunspots and subsequent coronal mass ejections (Owens and Crooker 2006). The second assumption concerns the rate at which heliospheric flux is lost. Observational evidence
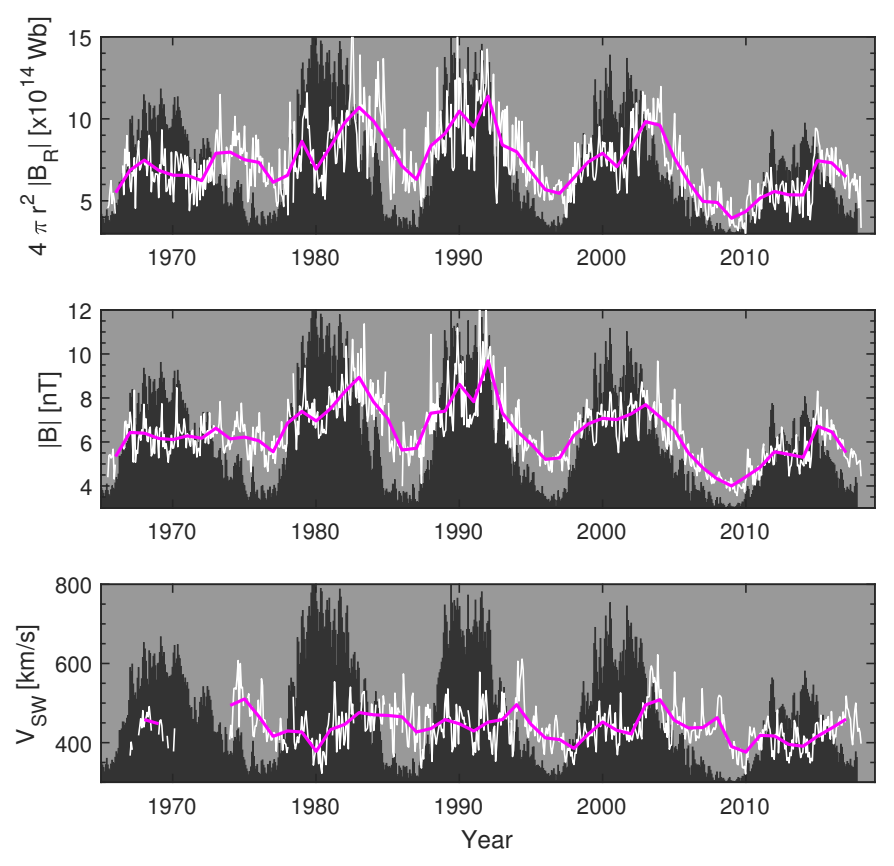

Figure 1. Near-Earth solar wind and heliospheric magnetic field variations over the space age. The dark-shaded region shows monthly sunspot number. White and magenta lines show 27-day and 1-year means of OMNI data, respectively. Panels, from top to bottom, show an estimate of total HMF (i.e., $4 \pi r^{2}\left|B_{R}\right|$, where $r=1 A U$ and $\left|B_{R}\right|$ is the modulus of the radial HMF component computed on 1-day data), the HMF intensity, $|B|$, and the solar wind speed, $V_{S W}$. 
(Sheeley Jr et al. 2001) suggests heliospheric flux loss rate varies with the inclination of the heliospheric current sheet to the solar rotational equator (Owens and Lockwood 2012), which approximately repeats each solar cycle. Thus sunspot number alone can be used to reconstruct the total HMF, shown as the red line in Figure 2.

Before 1610, reconstructions must be based on information stored in natural reservoirs, specifically concentrations of ${ }^{10} \mathrm{Be}$ in ice sheets and ${ }^{14} \mathrm{C}$ in ancient trees. These radionuclides are produced by spallation of galactic cosmic rays (GCRs) in the atmosphere (Usoskin 2017). As GCR intensity at the top of the atmosphere is determined by the level of heliospheric modulation, this provides a means of inferring the HMF over millennia. A number of intermediate steps are required, including knowledge of the interstellar spectrum, modelling the climate system (the carbon cycle, in the case of ${ }^{14} \mathrm{C}$, and deposition by precipitation, in the case of ${ }^{10} \mathrm{Be}$ ) and reconstruction of the modulation provided by the geomagnetic field. The inherent noise in the data and the time constants involved in the climate system mean that such reconstructions are limited to time scales of the order of 20-50 years, and thus cannot resolve individual solar cycles (however $M c$ Cracken and Beer (2015) have produced annual ${ }^{10}$ Be-based estimates for 600 years). After 1900, the data are contaminated by the Suess effects (caused by anthropogenic release of carbon from fossil fuels), and, later, atmospheric nuclear testing, making their interpretation in terms of solar variability difficult (e.g., Usoskin 2017, and references

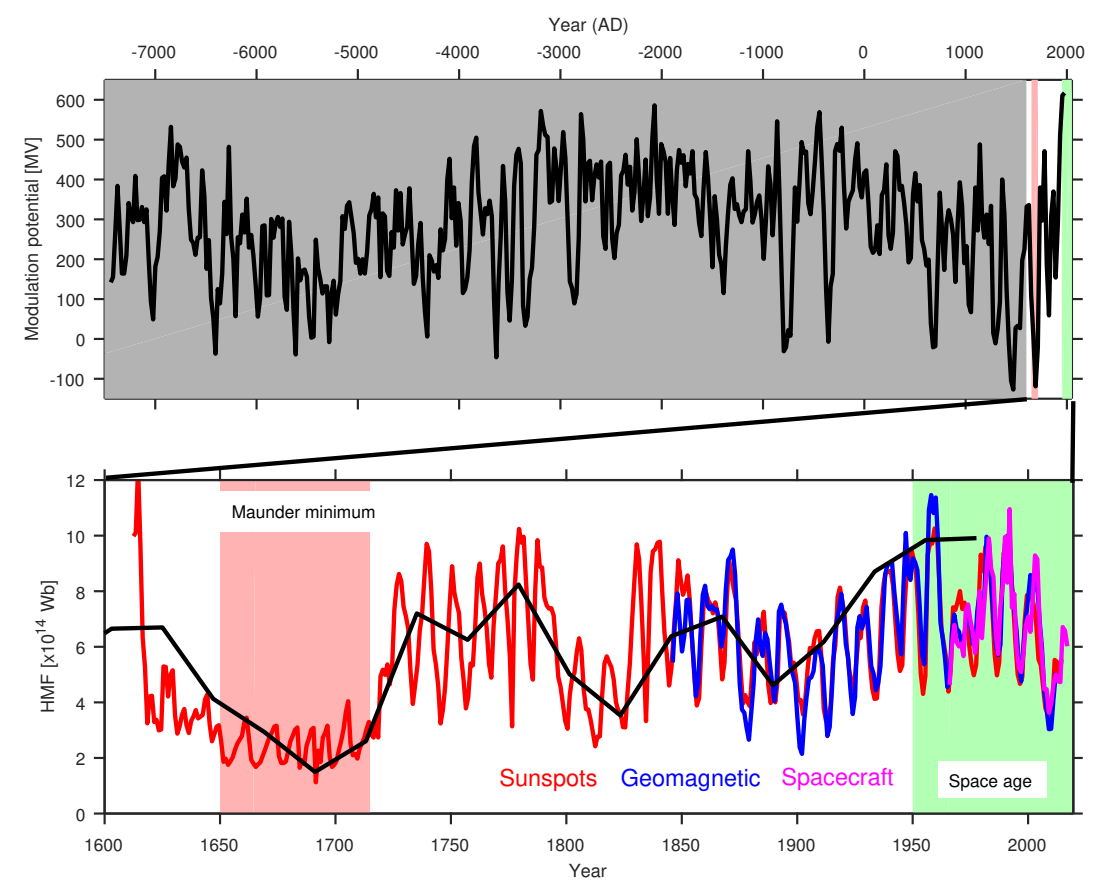

Figure 2. HMF variations over the centuries and millennia. The approximate span of the space age is shaded green, while the Maunder minimum is shaded red. The top panel shows the heliospheric modulation potential (HMP) over the last 9,400 years inferred from cosmogenic radionuclide records in tree trunks and ice sheets. This property is a proxy for the total heliospheric magnetic flux. Bottom: Heliospheric magnetic flux over the last 400 years from spacecraft observations (magenta), and geomagnetic- (blue), sunspot- (red) and cosmogenic isotope- (black) based reconstructions. 
therein). The reconstructed total HMF (Steinhilber et al. 2012), in the form of heliospheric modulation potential, is shown by the black lines in 2. Modulation potential has been linearly scaled in the bottom panel to provide a match to the HMF reconstructions. The variation over the last 400 years agrees well with the sunspot and geomagnetic reconstructions, particularly the timing and relative amplitudes of the Maunder minimum (1650-1715), Dalton minimum (1790-1820) and the modern grand maximum (1950-2010). The top panel shows that the space age has been a period of particularly high activity, and there have been a number of episodes of "grand minima" in the last 9400 years.

\section{Solar wind reconstructions}

Heliospheric reconstructions have focused primarily on HMF. Both geomagnetic and sunspot reconstructions, however, can also be exploited to estimate solar wind speed (Lockwood et al. 2014; Owens et al. 2017). Over the last 250 years, annual means of observations and reconstructions in near-Earth space, as shown by the coloured lines in Figure 3, have shown remarkably little variability, with average speeds of around 370-430 $\mathrm{km} \mathrm{s}^{-1}$ during both solar maximum and solar minimum. Globally (at all heliographic latitudes), the variation is very different, with mean speeds around $600 \mathrm{~km} \mathrm{~s}^{-1}$ at solar minimum, dropping to $400 \mathrm{~km} \mathrm{~s}^{-1}$ at solar maximum. During the Maunder minimum, however, both the near-Earth and global solar wind speed dropped below $330 \mathrm{~km} \mathrm{~s}^{-1}$.

\section{Summary}

Direct spacecraft measurements of the near-Earth heliospheric magnetic field (HMF) and solar wind extend back approximately 60 years. Prior to that, geomagnetic records can be used to produce accurate reconstructions of the annual near-Earth HMF intensity, $|B|$, and solar wind speed $\left(V_{S W}\right)$ back to 1845 . Assuming a Parker spiral HMF configuration and the observed heliolatitude constancy of the radial magnetic field component (Smith and Balogh 1995), these local properties can be used to estimate the global properties of total heliospheric magnetic flux (which is, in turn, a proxy for the open solar flux). Sunspot records can also be used as a proxy for the HMF production rate, allowing the annual HMF to be reconstructed back to 1610. Of course, the accuracy of this method is contingent on the accuracy of the underlying sunspot record(s), which are currently being revisited (e.g., Clette et al. 2014). Nevertheless, there is strong agreement with the

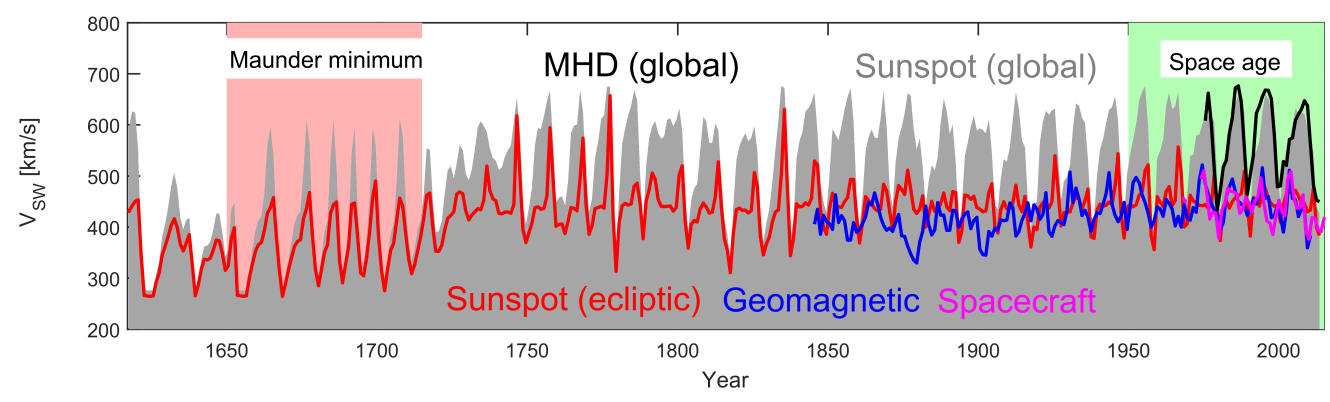

Figure 3. Solar wind speed $\left(V_{S W}\right)$ variations over the last 400 years. Coloured lines show annual means of near-Earth $V_{S W}$ from spacecraft (magenta), and geomagnetic- (blue) and sunspot(red) based reconstructions. The grey-shaded region and black line show global average $V_{S W}$ from magnetogram-constrained MHD simulations and sunspot-based reconstructions, respectively. 
geomagnetic HMF reconstructions over the 160 years of overlap. The largest discrepancy is for solar cycle 20, when spacecraft and geomagnetic reconstructions show little solar cycle variation in HMF, but sunspot number shows a modest variation. This difference is not well understood and is unlikely to be the result of uncertainty in either dataset. The most probable explanation is that the sunspot reconstructions are inherently global, while spacecraft and geomagnetic measurements are localised to near-Earth space.

In the 400-year reconstruction, the approximately 11-year solar cycle is the most prominent variation. Longer-term trends are also evident, most obviously the deep Maunder minimum (approximately 1650-1715), and the shallower Dalton minimum (approximately 1780-1820). A shallow minimum in the HMF occurred around 1900, followed by a rise until the 1980's (briefly interrupted by the peculiar solar cycle 20 in the 1960's). Since the 1980's, the HMF has been declining and at last solar maximum (approximately 20122014) was roughly equivalent to the small cycle around 1905.

Cosmogenic radionuclide data (i.e., ${ }^{14} \mathrm{C}$ and ${ }^{10} \mathrm{Be}$ from trees and ice sheets, respectively) allow the low-frequency variation of the HMF to be reconstructed. The Maunder, Dalton and 1900 minima are all well reproduced, as is the early 20th century rise. Looking over the full 9,400 years of reconstructed HMF, the 1600-present interval covered by the sunspot records exhibits the full range of variability (Acero et al. 2018) and thus can be used in the construction of a climatology. Conversely, geomagnetic, and particularly spacecraft observations, do not include the Maunder or even Dalton minima, and thus should not be used as a climatology of HMF variability (Henley and Pope 2017).

In the ecliptic plane over the last 250 years, there has been little variation in annual solar wind speed, $V_{S W}$. This is a result of the Earth sampling both fast and slow wind each solar rotation. At solar minimum and during the declining phase of the cycle, this is due to the inclination of the solar-flow band with respect to the solar rotation direction, allowing fast wind associated with polar coronal holes to enter the ecliptic (Gosling et al. 1995). At solar maximum, the faster in-ecliptic flows come instead from fast interplanetary coronal mass ejections or transient equatorial coronal holes. Globally, greater solar-cycle variation is observed, with peak speeds occurring at solar minimum when the polar coronal holes and associated fast wind are largest. Prior to 1750 , lower $V_{S W}$ is predicted both inecliptic and globally due to the reduced open solar flux and hence dearth of fast wind. The reduced global $V_{S W}$ and HMF is expected to reduce the size of the heliosphere and increase the average size of the Earth's magnetosphere (Owens et al. 2017).

Finally, we note that space climate reconstructions potentially provide probabilistic information about space weather. Analysis of geomagnetic records has revealed a strong correlation between annual means of geomagnetic activity and the number of moderate storms in a given year (Lockwood et al. 2018). This has been exploited with the sunspotbased HMF and $V_{S W}$ reconstructions (Owens et al. 2017) to quantify the likely decline in the occurrence of moderate space weather events under Maunder and Dalton minimumlike conditions. Of course, extreme space weather events are more poorly correlated with bulk of the distribution and thus may not show the same trends (Riley 2012).

\section{Acknowledgements}

M.O., L.B. and M.L. are funded by Science and Technology Facilities Council (STFC) grant ST/M000885/1.

\section{References}


Acero, F. J. et al., Extreme value theory applied to the millennial sunspot number series, Astrophys. J., 853(1), 80, 2018.

Clette, F. et al., Revisiting the sunspot number, Space Sci. Rev., 186(1-4), 35-103, doi:10.1007/s11214-014-0074-2, 2014.

Gosling, J. T., et al., The band of solar wind variability at low heliographic latitudes near solar activity minimum, Geophys. Res. Lett., 22(23), 3329-3332, doi:10.1029/95GL02163, 1995.

Henley, E. M., and E. C. D. Pope, Cost-loss analysis of ensemble solar wind forecasting, Space Weather, 15(12), 1562-1566, doi:10.1002/2017SW001758, 2017SW001758, 2017.

King, J. H., and N. E. Papitashvili, Solar wind spatial scales in and comparisons of hourly Wind and ACE plasma and magnetic field data, J. Geophys. Res., 110, doi:10.1029/2004JA010649, 2005.

Krivova, N. A. et al., Reconstruction of solar total irradiance since 1700 from the surface magnetic flux, Astron. Astrophys., 467, 335-346, doi:10.1051/0004-6361:20066725, 2007.

Lockwood, M., Reconstruction and prediction of variations in the open solar magnetic flux and interplanetary conditions, Liv. Rev. Sol. Phys., 10, 4, doi:10.12942/lrsp-2013-4, 2013.

Lockwood, M. et al., Reconstruction of geomagnetic activity and near-earth interplanetary conditions over the past $167 \mathrm{yr}$ - part 4: Near-earth solar wind speed, IMF, and open solar flux, Ann. Geophys., 32, 383-399, doi:10.5194/angeo-32-383-2014, 2014.

Lockwood, M. et al., Space climate and space weather over the past 400years: 2. proxy indicators of geomagnetic storm and substorm occurrence, Space Weather Space Clim., 8, A12, doi:10.1051/swsc/2017048, 2018.

McComas, D. J. et al., The three-dimensional solar wind around solar maximum, Geophys. Res. Lett., 30, doi:10.1029/2003GL017136, 2003.

McCracken, K., and J. Beer, The annual cosmic-radiation intensities 1391-2014; the annual heliospheric magnetic field strengths 1391- 1983, Sol. Phys., 290(10), 3051-3069, doi:10.1007/s11207-015-0777-x, 2015.

Owens, M. J., and N. U. Crooker, Coronal mass ejections and magnetic flux buildup in the heliosphere, J. Geophys. Res., 111(A10), A10,104, doi:10.1029/2006JA011641, 2006.

Owens, M. J., and R. J. Forsyth, The heliospheric magnetic field, Liv. Rev. Sol. Phys., 10, 5, doi:10.12942/lrsp-2013-5, 2013.

Owens, M. J., and M. Lockwood, Cyclic loss of open solar flux since 1868: The link to heliospheric current sheet tilt and implications for the maunder minimum, J. Geophys. Res., 117(A4), A04,102, doi:10.1029/2011JA017193, 2012.

Owens, M. J. et al., Estimating total heliospheric magnetic flux from single-point in situ measurements, J. Geophys. Res., 113, doi:10.1029/2008JA013677, 2008.

Owens, M. J., M. Lockwood, and P. Riley, Global solar wind variations over the last four centuries, Scientific Reports, 7, 41,548, doi:10.1038/srep41548, 2017.

Owens, M. J., et al., Near-earth heliospheric magnetic field intensity since 1750 . part 1: Sunspot and geomagnetic reconstructions, J. Geophys. Res., 121, 6048-6063, doi:10.1002/2016JA02252, 2016 .

Riley, P., On the probability of occurrence of extreme space weather events, Space Weather, 10(2), n/a-n/a, doi:10.1029/2011SW000734, s02012, 2012.

Sheeley Jr, N. et al., Coronal inflows and the sun's nonaxisymmetric open flux, Astrophys. J. Lett., 546(2), L131, 2001.

Smith, E. J., and A. Balogh, Ulysses observations of the radial magnetic field, Geophys. Res. Lett., 22, 3317-3320, doi:10.1029/95GL02826, 1995.

Solanki, S. et al, Secular variation of the sun's magnetic flux, Astron. Astrophys., 383(2), 706$712,2002$.

Steinhilber, F., et al., 9,400 years of cosmic radiation and solar activity from ice cores and tree rings, Proc. Nat. Academy of Sci., 109, 5967-5971, doi:10.1073/pnas.1118965109, 2012.

Svalgaard, L., and E. W. Cliver, The idv index: Its derivation and use in inferring long-term variations of the interplanetary magnetic field strength, J. Geophys. Res., 110, 12,103-+, doi:10.1029/2005JA011203, 2005.

Usoskin, I. G., A history of solar activity over millennia, Liv. Rev. Sol. Phys., 14(3), doi:10.1007/s41116-017-0006-9, 2017.

Vasyliunas, V. M. et al., Scaling relations governing magnetospheric energy transfer, Planet. Space Sci., 30(4), 359-365, doi:10.1016/0032-0633(82)90041-1, 1982. 